\title{
Attribution analysis of high precipitation events in summer in England and Wales over the last decade
}

\author{
Friederike E. L. Otto • Suzanne M. Rosier • \\ Myles R. Allen • Neil R. Massey • Cameron J. Rye • \\ Jara Imbers Quintana
}

Received: 15 January 2013 / Accepted: 17 February 2014 / Published online: 11 October 2014

(C) Springer Science+Business Media Dordrecht 2014

\begin{abstract}
The crucial question in the public debate of extreme events is increasingly whether and to what extent the event has been caused by anthropogenic warming. In this study we investigate this question using extreme summer precipitation events in England and Wales as an example for probabilistic event attribution using very large ensembles of regional climate model (RCM) simulations within the weather@home.net project. This allows us to analyse the statistics of high precipitation events in England and Wales, a region with a high quality precipitation observational dataset. Validating the model simulations against observations shows a credible shape of the distribution of 5-day precipitation, and thus confidence in the results. While the risk of extreme July precipitation events has at least doubled due to anthropogenic climate change in the modelling framework, no significant changes can be detected for the other two summer months. This study thus highlights the challenges of probabilistic event attribution of complex weather events and identifies
\end{abstract}

Part of the EQUIP special issue of Climatic Change

This article is part of a Special Issue on "Managing Uncertainty in Predictions of Climate and Its Impacts" edited by Andrew Challinor and Chris Ferro.

F. E. L. Otto $(\bowtie)$

Environmental Change Institute, University of Oxford, South Parks Road, Oxford, OX1 3QY, UK

e-mail: friederike.otto@ouce.ox.ac.uk

M. R. Allen · N. R. Massey

Environmental Change Institute, University of Oxford, Oxford University Centre for the Environment, South Parks Road, Oxford, OX1 3QY, UK

M. R. Allen · C. J. Rye · J. I. Quintana

Atmospheric, Oceanic and Planetary Physics, University of Oxford,

Parks Road, Oxford, OX1 3PU, UK

Present Address:

S. M. Rosier

National Institute of Water and Atmospheric Research Ltd.,

301, Evans Bay Parade, Wellington 6021, New Zealand 
the need to further decompose atmospheric features responsible for an event to occur for quantitative attribution analysis.

\section{Introduction}

In recent years wet British summers have been reported frequently, with record-breaking rainfall in June in 2012 and a wet July following a wet spring, and the exceptionally rainy July in 2007 , not record breaking in the monthly totals but with very intense shorter rain events within the month, which resulted in heavy summer floods in middle England and Wales. Although large scale flooding held off in 2012, the extreme rain caused damage to the harvest and put strain on infrastructure systems. However the heavy flooding in 2007, following very high daily and 5-day totals (with 3 top 12 5-day totals in July compared to the whole observed period, and the 5th highest total daily precipitation), was classified as a national disaster by the Environment Agency causing economic losses of approximately $£ 3$ billion (Report Environment Agency UK, 2010). There is no significant trend in July rainfall extremes, however, as extremes are by definition rare, a trend would only be detectable if the risk of extremes changed dramatically. Trend analysis will thus not reveal subtle changes in extreme precipitation. In times of anthropogenic climate change with increasing global mean temperatures the question arises if the observed extreme events were just bad luck or whether heavy rainfall in summer, especially in July, is something we have reasons to expect in the future and should begin adapting to in terms of infrastructure planning? Even before a trend might be detectable, attribution analysis can reveal the influence of anthropogenic climate change on very rare events, and such is the aim of this paper. July precipitation on a timescale relevant for flooding will be the focus of this study. It is important to highlight here that precipitation in the relevant month, although important, is only one factor determining flood risk (Kay et al. 2011). We attempt to answer the question of how the odds of extreme July precipitation relevant for flooding have changed, not the odds of floods occurring in July. Increasingly, politicians, decision-makers and non-governmental organisations (NGOs) want to know whether and to what extent global human-influenced climate change is affecting localised extreme weather events, highlighted by the fact that the Conference of the Party (COP) meeting in Doha in November 2012 established a work programme specifically on loss and damage due to climate change (UNFCCC report FCCC/SBI/2012/29 2012), further developed into the "Warsaw mechanism" at the COP in 2013. Studies into the European heat wave of 2003 (Stott et al. 2004), the England and Wales floods of 2000 (Pall et al. 2011), and the Russian heat wave of 2010 (Dole et al. 2011; Rahmstorf and Coumou 2011; Otto et al. 2012) have sought to determine to what extent the risks of these events occurring have changed because of anthropogenic greenhouse gas emissions, many of them using the emerging method of probabilistic event attribution (PEA). Under the assumption of an unchanging relationship between hazard and resulting damage, event probability can be seen as a proxy for risk (Pall et al. 2011). One of the most recent series of studies in this field (Peterson et al. 2012; Peterson et al. 2013) attempts to answer this question for several extreme events that occurred in 2011 and 2012, with one of the studies being an attribution study of exceptionally warm Novembers in central England (Massey et al. 2012), which explicitly applies PEA. It finds a 40 times increase in the risk of such warm Novembers occurring, when comparing simulated temperatures of the 2000s with the 1960s. Pall et al. (2011) compare river runoff derived from daily precipitation in the autumn of 2000, with an autumn 2000 in a world that might have been without anthropogenic greenhouse gas forcing and associated sea surface temperature warming. In our study we use a 
PEA approach combining both methods, as outlined in the following Section 2. In Section 3 the results are presented and analysed with emphasis on the quantification of uncertainty (see Section 2.4) and concluding remarks are made in Section 4.

\section{Methodology}

The aim of this study is to use an approach of probabilistic event attribution (PEA) to investigate whether the odds of extreme precipitation in July, given the boundary conditions of observed forcings and sea surface temperatures (SSTs), have changed due to anthropogenic influences, making use of very large ensembles of regional climate models.

\subsection{Probabilistic event attribution (PEA)}

To achieve our aim of a probabilistic event attribution study of extreme July precipitation in England and Wales (such as led to severe flooding in large parts of central England, Northeast England and Wales), at least two climate model ensembles are required. The first one is a historical ensemble using observed forcings and sea surface temperatures (SSTs), while the second is a counterfactual ensemble for the "world that might have been" without anthropogenic forcing. Both ensembles must be sufficiently large to ensure that the statistical analysis of changes in extreme events is robust (e.g., Pall et al. 2011; Kay et al. 2011). Some authors have also compared the change in event occurrence in model simulations between a recent decade (e.g., 2000s) and an earlier decade when the anthropogenic forcing was not as strong (e.g., 1960s) (e.g., Otto et al. 2012; Massey et al. 2012). Both approaches have their advantages and disadvantages. A comparison of the 1960s with the 2000s does not allow for the changes in probability of occurrence of extreme events to be attributed to anthropogenic climate change alone as other climate conditions have been different in the two decades as well. It does, however, allow for a validation of modelling results against observations. Additionally comparing whole decades instead of ensembles of single years minimises the influence of large scale teleconnection patterns, e.g. the North Atlantic Oscillation (NAO), as decadal ensembles smooth interannual variability of such oscillations. However, this does not apply for the Atlantic Multidecadal Oscillation (AMO) pattern which switches modes only on decadal and longer timescales (Sutton and Dong 2012). Especially with respect to precipitation, which is resolvable for large scales but largely either not understood or not resolvable even in a regional climate model on local scales that are important for flooding, both aspects - the ability to validate the model and the reduced influence of specific SST patterns - are important.

\subsection{Modelling analysis}

Assessing the influence of external drivers (e.g., increased greenhouse gas concentrations in the atmosphere) on extreme weather is challenging because the most important events are typically rare, so their observed frequency is dominated by chance. In order to compile robust statistics of extreme weather events, large ensembles of model simulations at relatively high resolution are required. This project makes use of the large-ensemble capability provided by the on-going climateprediction.net 'weather@home' volunteer computing network (Allen 1999; Massey et al. 2006), where members of the public are producing multi-thousand-member ensembles of weather simulations using regional climate models (RCMs) of different parts of the world. We have applied the model set-up described in 
Massey et al. (2012) with the regional climate model (RCM) embedded within a general circulation model (GCM). The increased resolution of the RCM results in a more realistic simulation of localised weather events, including high and low temperatures and extreme precipitation over a relatively small area (Jones et al. 2004). The standard ensemble described below is an initial condition ensemble hindcast experiment over Europe which simulates the historical period 1960-2010 including all observed forcings (AF)(we will only analyse the 1960s (AF1960s) and 2000s (AF2000s) in this study) as well as the decade from 2000 to 2010 with the anthropogenic climate change signal removed (NAT2000s) as described in Section 2.3. For the results used in this study, the RCMs run by volunteers are at $50 \mathrm{~km}$ resolution over Europe driven by a global atmospheric model. This is a relatively low resolution for an RCM but given that natural variability is the largest source of uncertainty (Section 2.4) the best methodology to account for this uncertainty (given resources are not unlimited) is to trade accuracy for precision and employ large ensembles of relatively low resolution. The models used are HadAM3P, an atmosphere only GCM at $1.25 \times 1.875$ degrees resolution, forced with observed SSTs from the HadISST data set (Rayner et al. 2003) and the RCM HadRM3P. Both models have been developed by the UK Met Office and are based upon the atmospheric component of HadCM3 (Pope et al. 2000; Gordon et al. 2000) with some improvements in the model physics described in Massey et al. (2012). Both models are run many hundreds of times with varied initial conditions. In this way, very large ensembles of RCM simulations can be computed, of the order of thousands, which in turn allows greater confidence when examining the statistics of rare events. We follow a similar methodology to Massey et al. (2012), which uses very large ensembles of general circulation models (GCMs) to assess the change in risk of very warm Novembers in central England under two different climate scenarios: observed July in the decade between 1960 and 1970 and observed July between 2000 and 2010. In addition to that we analyse the same decade, 2000-2010, in a representation of a world that might have been without anthropogenic climate change.

Although our method of comparing the 1960s with the 2000s does not permit clean decoupling of anthropogenic and natural drivers, using decadal long scenarios of precipitation reduces some of the effects of natural variability and allows both scenarios to be validated against observed data. The main observational dataset used here is the HadEWP data which are part of HadUKP, the UK regional precipitation series provided by the Met Office Hadley Centre (Alexander and Jones 2001). As well as the analysis of large ensembles of at least two scenarios showing different climate conditions, the other crucial component of an event attribution study is the validation of the model, investigating whether it is capable of representing the extreme event.

Figure 1 shows quantile-quantile plots as a measure of the model's ability to represent the observed distribution of precipitation. Both model (HadRM3P) and observational (HadEWP) data are shown in the two different decades,daily averages as well as the 5day average computed as a running 5-day mean are shown. Pall et al. (2011) showed that daily means are the important timescales to derive river run-off,however, if river run-off is not being computed, 5-day means are a better proxy for flood risk than daily means, giving some indication of ground saturation and maintaining sub-monthly variability. Fowler and Kilsby (2003) demonstrated that multi-day precipitation is an important causal factor in floods, and found no significant changes in 1-2 day precipitation in the period 19912000 compared to earlier decades but significant changes in 5-day events. Furthermore a relatively low resolution RCM is probably more reliable on 5-day timescales as very local mechanisms accounting for extreme changes in daily precipitation are generally not well represented by these types of models, especially in the summer months. Fowler et al. 

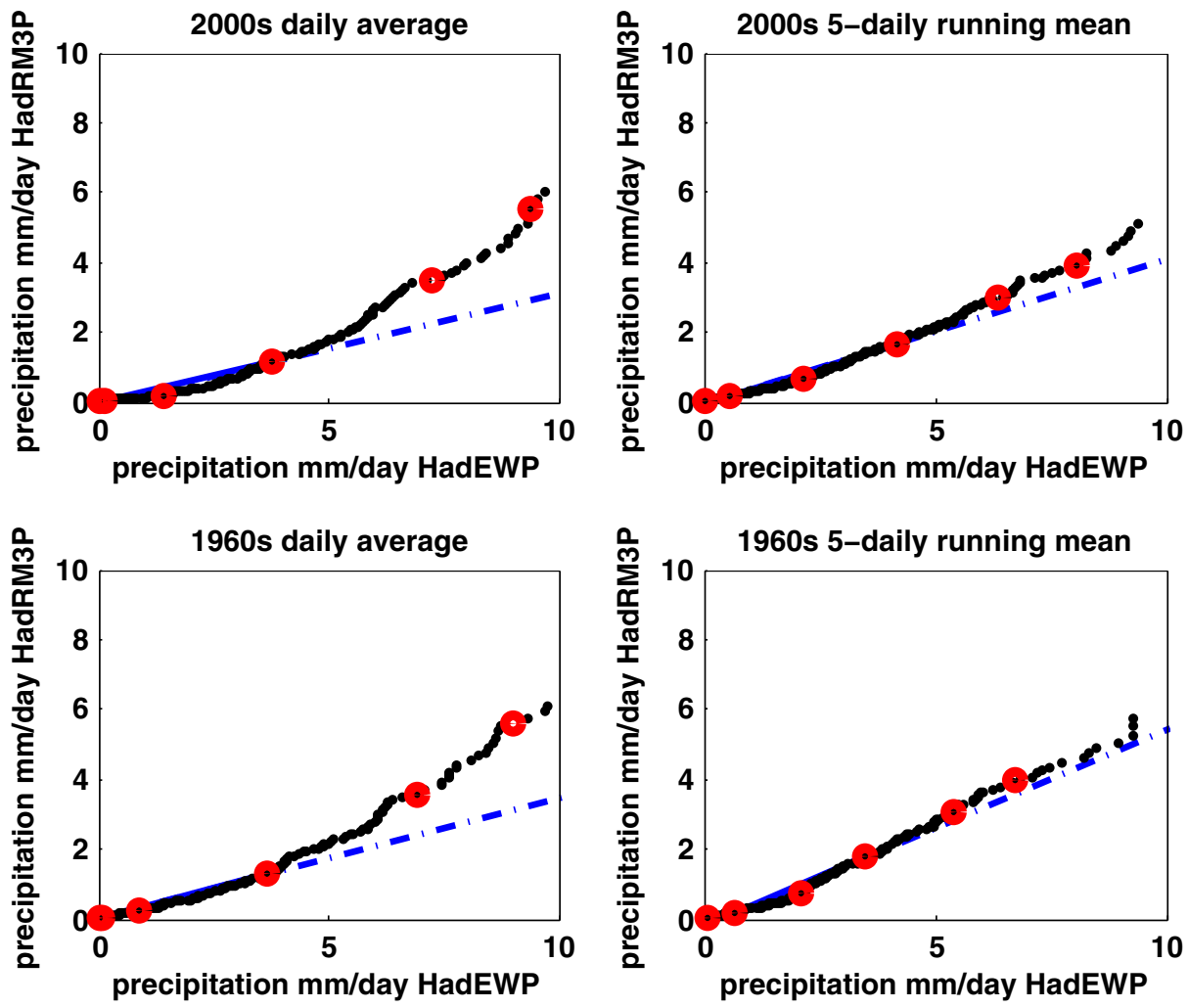

Fig. 1 Quantile-quantile plots of observed (HadEWP) and modelled (HadRM3P) daily (left) and 5-day (right) July precipitation in England and Wales in the decade from 2001-2010 (top) and 1961-1970 (bottom). The red dots represent, from left to right, the 5, 25, 50, 75 and $95 \%$-quantiles

(2007) demonstrated that RCMs are in general more likely to reproduce the observed distribution of 5-day events compared to daily precipitation, an analysis our model validation below supports. The observed data set used for validation gives the area average over England and Wales encompassing roughly a region from $51.3-53.2 \mathrm{~N}$ and $356.2-0.3 \mathrm{E}$. This region (51.3-53.2N, 356.2-0.3E) both encompasses the regions of highest flooding in 2007 in Central and North East England and is large enough to be expected to be represented well in an RCM of this resolution. From this figure and complementary reliability diagrams (not shown) it becomes evident that the model underestimates the observed precipitation, and that the distribution is quite well modelled within the lowest $50 \%$-quantile, but allocates insufficient probability to the upper percentiles of the distribution. The straight line in Fig. 1 is the linear fit between both distributions, if both lay on this line the distributions would be identical. The fact that this line is not the 1-1 line shows that the magnitude of precipitation in the model does not match observations. Comparing 5-day means of precipitation as shown on the right-hand side of Fig. 1 reveals a much improved goodness-of-fit even at the wet tail of the distribution which is important with respect to extreme precipitation events, but also highlights that the magnitude of precipitation is underestimated in the model. Attempting to counteract the bias in magnitude by simply multiplying the mean 
difference leads to better reliability of the overall mean but does not improve the fit at the tails of the distribution. We thus refrain from applying a bias correction as we are only comparing the model with the model so the actual magnitude is irrelevant for the analysis. We concentrate our analysis on 5-day means which give the better distribution in the model and are furthermore an important indicator for flood risk (e.g, Fowler and Kilsby 2003; Fowler et al. 2010). Additionally, the underestimation of the observations is consistent for the 1960s and the 2000s so any changes in frequency and magnitude between the two decades represents a change in the relative risk of extreme precipitation.

\subsection{Modelling "the world that might have been"}

The natural-only forcing used to simulate a counterfactual ensemble of the decade 20012010 is a climate forcing that is identical to the one we observed in the last decade but with greenhouse gas emissions, ozone, $\mathrm{SO}_{2}$ and DMS held at preindustrial levels, and with the corresponding lower SSTs prescribed. It thus simulates a world that might have been without anthropogenic influences.

The attributable twentieth-century greenhouse gas warming in SSTs cannot be found directly from observations, because observations also contain the signal of both natural (e.g., solar and volcanic) and other anthropogenic (e.g. sulphate aerosol) drivers, and internal variability. Prior studies (Stott et al. 2006) derived this warming from estimates that used established 'optimal fingerprinting' analysis (Hegerl et al. 2007; Stott et al. 2010), which uses multiple linear regression to compare observed surface temperature change and is described in the supplementary text of Pall et al. (2011).

We use a new method based on Pall et al. (2011) in which we subtract warming patterns from observed SSTs. The Met Office state-of-the-art coupled climate model HadGEM2 is used to compute delta values (SST difference fields) by subtracting SSTs of a HadGEM2 'natural' run over the last decade without anthropogenic greenhouse gas, ozone, $\mathrm{SO}_{2}$ and DMS forcing from the same model runs with 'all forcing'. These delta SSTs are then subtracted from HadISST SSTs. To reduce noise, the deltas are produced using decadal averages. The counterfactual SSTs were then used to estimate the sea ice concentration for the 'world that might have been'. As current state of the art GCM sea ice projections are inconsistent with observations and with respect to other models (Eisenmann et al. 2007), we apply an empirically-based method (Rayner et al. 2003) to provide sea ice fields for the natural model runs. This method to generate possible sea ice fields is independent of GCMs, using a statistical method to fit a quadratic equation to SSTs. Using observational records from HadISST SSTs (SST) and sea ice extent (SIE) the first order approximation:

$$
S S T=a \times S I E^{2}+b \times S I E+c
$$

is derived. Under the assumption that this approximation holds on the time scale of interest so that the parameters $a, b$, and $c$ are time invariant, the sea ice extent (SIE) for given SSTs can be calculated. To test the algorithm this method is used to calculate the observed SIE for the period 1999-2010. Figure 2 shows the result of this validation as well as the sea ice extent for the natural climate simulations for the same period and the algorithm is seen to perform well. 


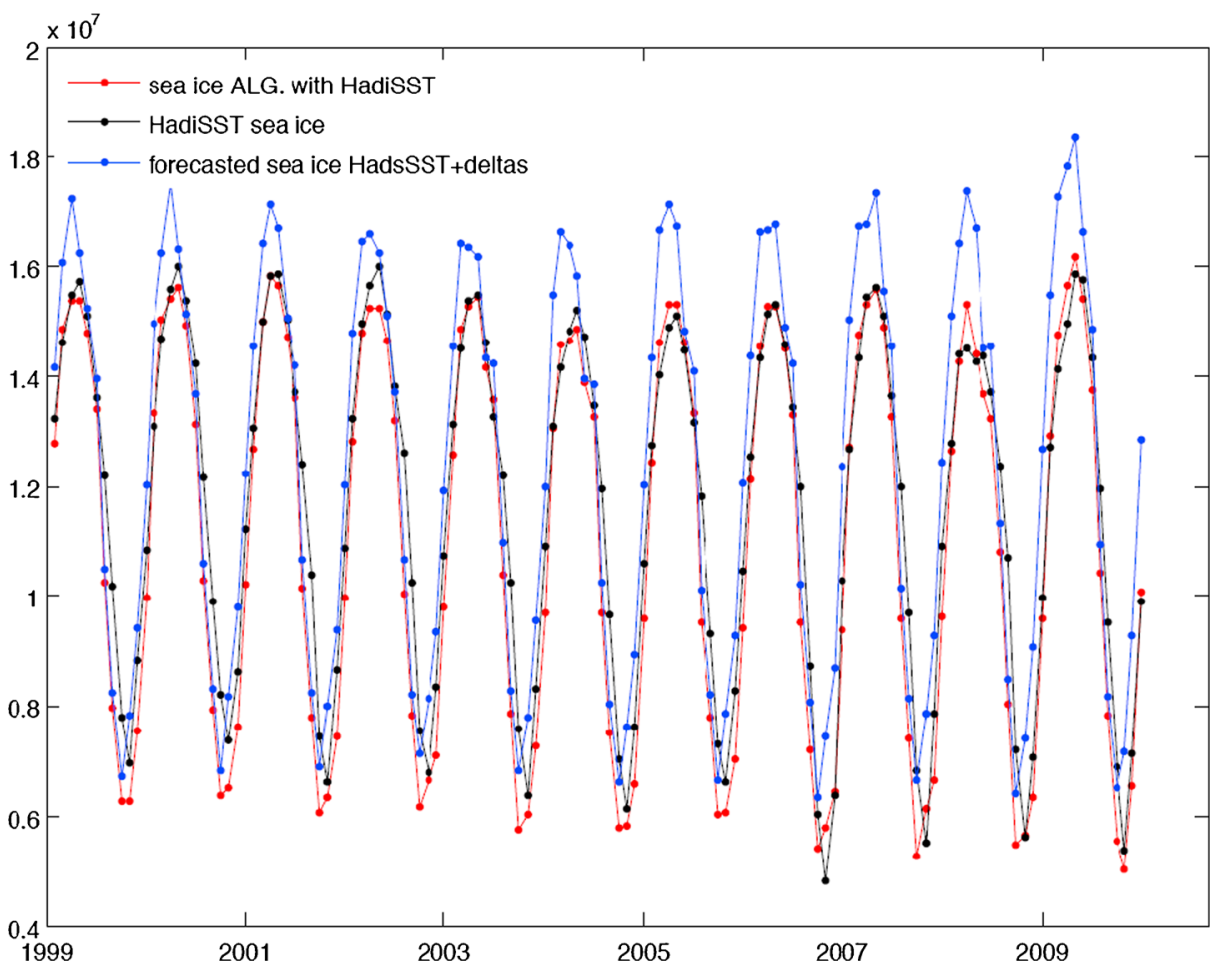

Fig. 2 Sea ice extent from 1999-2010 in observed HadISST data (black), from the sea ice algorithm (ALG, red), and in the "world that might have been" (blue)

\subsection{Uncertainty assessment}

The main source of uncertainty in the regional precipitation in present day climate is the natural variability (Hawkins and Sutton 2011). The modelling framework employed in this study is ideal to quantify this uncertainty by using a very large ensemble of simulations of the very same climate conditions with slightly varied initial conditions. However, another large uncertainty, the modelling uncertainty, cannot be addressed as only one model is used for the study. Validating the model against observations gives insights into the shortcomings of the model but cannot quantify this uncertainty. Introducing observations into the experiment reveals another source of uncertainty - the uncertainty within the observations. However, as observed data sets in this part of the world are fairly consistent, we assume this to be a small part of the overall uncertainty. To validate the model's ability to reproduce observations, we compare model precipitation with the observed timeseries from HadEWP, making use of quantile-quantile plots and reliability diagrams (not shown) as statistical validation tools, described in Section 2.2. England and Wales have long records of observed precipitation, and a very dense network of weather stations. Therefore we might expect the uncertainty in this observed dataset to be somewhat smaller than in most other regions. For a qualitative assessment of uncertainty in the observed data we additionally compute quantile-quantile plots for the decades of 2001-2010 and 1961-1970 using NCEP reanalyses and our model's data. NCEP is certainly not the ideal data set for regional precipitation but 
is one of the few spanning the considered timeframe 1960-2010. Furthermore it is derived very differently from HadEWP and is thus arguably suitable to check whether validation with very different data sets gives very different results, which turns out not to be the case. The picture (not shown) represents good agreement but with the 5-day mean data slightly overestimating the NCEP data in the higher quantiles. We made no attempt to quantify the uncertainty in the observed data sets, but assessments can be found in (Alexander and Jones 2001; Kalnay et al. 1996). It is important to note that we are not so interested in the model's ability to simulate the correct precipitation at the correct time but more to represent the right distribution of precipitation within the relevant decade. The validation analysis is thus done separately for the two analysed decades.

The model set-up we use for the first part of this study has been especially designed to account for uncertainty in the initial conditions input to the model. In order to produce a robust statistical analysis, we use an ensemble size of 1741 initial conditions. By current standards in this area of science this is a large ensemble - thus we believe we are able to sample the initial condition uncertainty exceptionally well. Using this standard ensemble we focus purely on the initial condition uncertainty - all other aspects, of model structure and external forcing, have not been varied at all. Therefore the uncertainties in other input variables cannot be quantified in this approach.

In the later part of the study, we examine differences between models representing conditions of the 1960s and models representing conditions of the 2000s with the anthropogenic signal in the SST forcing removed. The application of this method can give an initial bound on the uncertainty originating in the fact that we do not know how present day climate without anthropogenic emissions would be, but is likely to underestimate the full range. To make more comprehensive statements about the true range of uncertainty in this area, further studies should apply different warming patterns from different GCMs to remove the anthropogenic signal from the SSTs (such as was done by Pall et al 2011). The use of a regional climate model driven by a global model, and the consistency of the two models, allows us to gain confidence in the model physics on different scales, even though it does not allow us to quantify the uncertainty in these methods explicitly.

In this study we have not attempted to quantify any other type of modelling uncertainty, e.g. we have not varied any internal model parameters. All other sources of uncertainty affecting these results, e.g. stemming from stochastic variability and our own expert judgment and interpretation of results, cannot be accounted for in this approach.

\section{Attribution analysis}

To analyse the results for precipitation from the regional modelling experiment, first two separate ensembles are formed from the data replicating the spatial distribution of central England and Wales (51.3-53.2N, 356.2-0.3E). The ensembles are: all the Julys occurring in the 1960s and all Julys in the 2000s. Our initial analysis involves comparing the two large model ensembles that represent simulations of the 1960s (AF1960s) and 2000s (AF2000s) respectively. For each of these decades our ensemble is effectively an initial condition ensemble across all the years of that decade. Hence for each of the decades we examine a very large ensemble that represents many hundreds of different realisations of possible weather situations in that decade, given the climatological forcing conditions.

Additionally a third ensemble simulating the decade of 2001-2010 with the anthropogenic greenhouse gas signal removed (NAT2000s) is created. Figure 3 shows all three ensembles - 5-day average July precipitation in England and Wales is shown, for the 1960s 


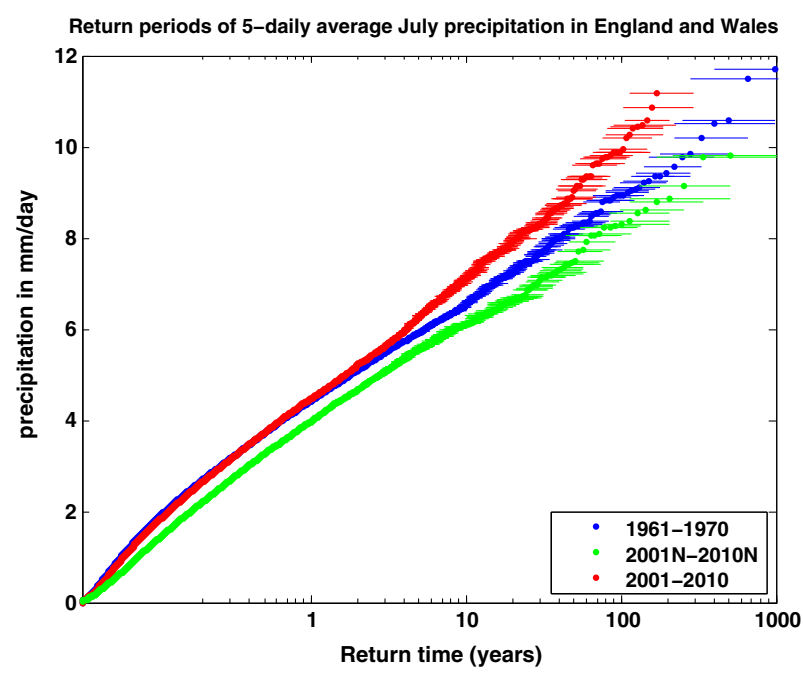

Fig. 3 Return times of 5-day mean precipitation in July in the 1960s (blue) and 2000s (red) as well as the natural 2000s (green). The error bars represent the 5-95\% confidence interval after resampling the ensemble, applying straightforward bootstrapping

(blue), the 2000s (red) and the counterfactual 2000s (green). A similar comparison is made for June and August (Fig. 4), which gives a different result which will be used to assess the reliability of the result, but our main focus is the July 5-day precipitation shown in Fig. 3.

The curves in Fig. 3 reveal very little difference between the 1960s and the 2000s over much of the lower (drier) end of the ensembles, but a small separation of these two curves towards the higher (wetter) end. Where the curves separate, it is the case that the extreme wet events become more likely (shorter return time) under the 2000s (AF2000s, red) conditions than in the 1960s (AF1960s, blue). This general result is in keeping with expectations from Clausius-Clapeyron constraints that, in a warmer climate (such as the 2000s here),
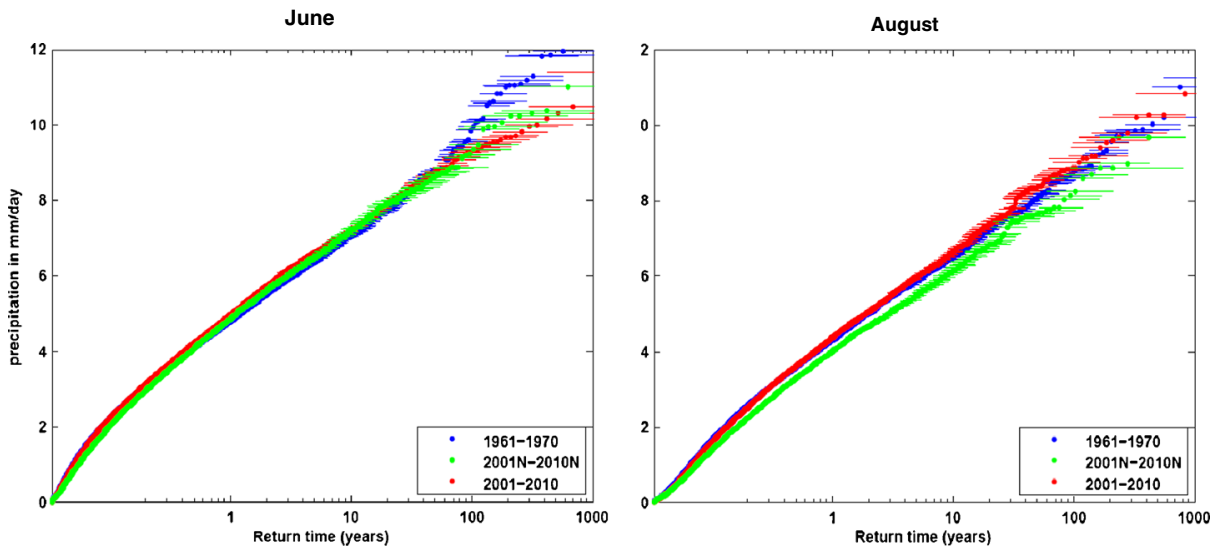

Fig. 4 Return times of 5-day mean precipitation in June (left) and August (right) in the 1960s (blue) and 2000 s (red) as well as the natural 2000s ( green). The error bars represent the 5-95\% confidence interval after resampling the ensemble, applying straightforward bootstrapping 
precipitation is expected to occur in higher-volume events. The counterfactual ensemble for the 2000s (NAT2000s, green) shows similar behaviour to the 1960s with the simulation of preindustrial precipitation being below the values of present day precipitation throughout. In all three ensembles the separation is small but significant especially comparing the NAT2000s ensemble with the all forcings simulation AF2000s.

This result is repeated but weaker, with less separation of the three curves, in identical ensembles for August (Fig. 4, right) but cannot be seen in return times of 5-day precipitation in June in the three ensembles (Fig. 4, left).

\subsection{Attributable risk}

Only by explicitly simulating climates including all possible weather states consistent with the climate forcing conditions for the period of interest can the uncertainties be addressed associated with the question: what fraction of the event probability is attributable to the anthropogenic drivers? The climate forcing conditions are simulated using observed greenhouse gas and aerosol forcings and observed SSTs. Thus not all possible climate states are simulated but possible weather given the historical SSTs. The large initial condition ensemble used addresses the uncertainty in the simulated weather to a high degree and under the assumption of an unchanging relationship between hazard and resulting damage, event probability can be seen as a proxy for risk (Pall et al. 2011). The return times of the 5-day means in Figs. 3 and 4 are almost straight, parallel, lines comparable to the return times of river runoff (Pall et al. 2011) which is in contrast to, e.g., monthly means or shorter timescales (Fowler et al. 2010). The straight parallel lines corroborate the finding of, e.g., Stott et al. (2004) and Allen et al. (2007) that for the fraction of attributable risk (FAR) calculation (Allen 2003), the actual value of the threshold does not matter. Therefore return times of precipitation based on 5-day means are a good measure for event attribution studies as a bias in magnitudes does not matter if the fractional change in return time is independent of the threshold. Our choice to concentrate on 5-day means is therefore not only adequate in terms of flood risk assessment but also for the independence of the threshold for risk attribution.

Results from the main analysis (Fig. 3) do show a significant change in the return times for a high precipitation event like that which caused the floods in 2007 . The highest 5-day mean in July 2007 was about $9 \mathrm{~mm}$ per day in the observations. However, as the model is biased this value is not directly applicable to the modelling results. In July, a one in 100 year event in the natural ensemble (NAT2000s) is a one in approximately 35 year event in the 2000s in present day conditions (AF2000s). At the same time a one in a 100 year event in the 1960s (AF1960s) has become approximately a one in 50 year event under present day climate conditions (AF2000s). Both ensembles, the historical and the counterfactual one (AF1960s, NAT2000s), represent possible ways to simulate a world without anthropogenic climate forcing; thus the spread between these two ensembles gives an estimate of the uncertainty in the anthropogenic climate change signal, but probably not the full range of uncertainty. The 1960s simulations already include some amount of anthropogenic greenhouse gases and aerosols - both types of emissions probably influence precipitation over the UK. Sparrow et al. (2013) show that the SST pattern obtained from the GCM HadGEM2 which is used in this experiment (NAT2000s) shows an SST pattern in the North Atlantic which is in contrast to similar GCM simulations with identical counterfactual climate forcings. The North Atlantic is an important region influencing the weather systems that bring rainfall over the British Isles. Further studies using a whole ensemble of SST patterns from different climate models will reveal a more comprehensive quantification of uncertainty in 
the anthropogenic signal on local weather events. The analysis in this study reveals that the risk of 2007 type July extreme precipitation has more than doubled due to anthropogenic climate change. The same cannot be said for summer precipitation as a whole as results for August and especially June are less clear (Fig. 4). For comparing our analysis with recently published analysis on very wet summers in Northern Europe (Sutton and Dong 2012) we additionally calculate the surface air temperature and precipitation anomalies for the two analysed decades (Fig. 5).

Recent analysis of several European extreme weather events (Coumou and Rahmstorf 2012), including the England and Wales floods of 2007, show that a general increase in such extreme weather events can be expected in a warming climate. This study, based on a model, is, for July, consistent with this and another recent study by Sutton and Dong (2012), who show an increase in wet summers over Northern Europe, including England and Wales, comparing two periods of high Atlantic Multidecadal Oscillation (AMO) (19311960, 1996-2009) with a period of low AMO index (1964-1993) in observations. Their interpretation suggests a causal relationship between the AMO index, surface air temperature (SAT) anomalies and precipitation over Europe, as well as mean sea level pressure (MSLP) over Europe and the North Atlantic. Since the two periods we investigate fall almost (we also analysed the correct phases) within the two contrasting AMO phases and our model is driven with observed SSTs, providing thus a realistic AMO, we calculate SAT and precipitation anomalies for the decade of the 2000s and the 1960s as well as in the two AMO periods (1964-1993,1996-2009) identified by Sutton and Dong. Figure 5 shows the resulting anomalies in the compared periods of the 1960s and 2000s. The anomalies for the periods 1964-1993 subtracted from 1996-2009 look very similar to the anomalies shown in Fig. 5. As causalities are clearer in a model, given that we understand the model mechanisms and feedbacks, similar results to Sutton and Dong would corroborate the assumption that AMO phases have an influence on wet British summers.

While the SAT anomalies are very similar to the findings of Sutton and Dong (2012) the anomalies for precipitation differ whereas the MSLP anomalies (not shown) are difficult to compare because the region analysed in Sutton and Dong (2012) does not match the region our regional model comprises. Positive MSLP anomalies are found over the British Isles in the model contrasting with negative anomalies in Sutton and Dong (2012). The precipitation anomalies in the model are much smaller than the observed anomalies in Sutton and Dong and while the pattern of increased precipitation over Scandinavia is comparable, there is no
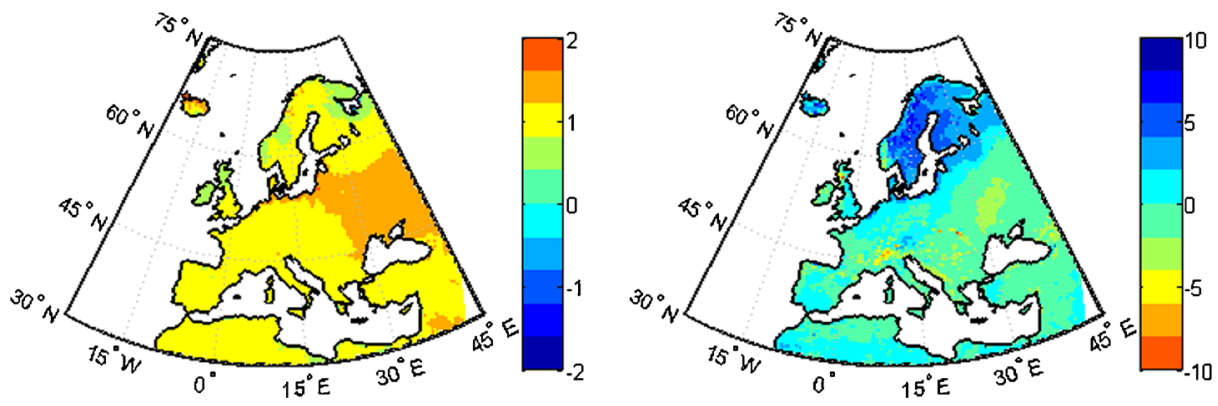

Fig. 5 Left: mean summer (JJA) surface air temperature anomalies (in degrees Celsius) over Europe, subtracting the decadal mean of the 1960s from the decadal mean of the 2000s. Right: mean summer (JJA) precipitation anomalies (in percent) over Europe, subtracting the decadal mean of the 1960s from the decadal mean of the $2000 \mathrm{~s}$ 
change in precipitation over the British Isles in the model which is again in contrast to Sutton and Dong (2012). Redoing this analysis for the identified AMO phases (not shown) gives very similar results, although the early 1960s are not representing an especially low AMO phase. Also comparing the $95 \%$-quantiles of precipitation rather than mean precipitation does not give qualitatively different results. A reason for this could be that the mean sea level pressure in the model is slightly overestimating the Azores High compared to Sutton and Dong (2012), which could result in an eastward shift of the pattern of increased precipitation in the model compared to the observed data. However, the overestimation is rather small compared to the ERA-interim reanalysis (not shown). The fact that we do not reproduce Sutton and Dong's results could mean that our model is inaccurate, or that the AMO is not the most important trigger of increased summer precipitation over England and Wales.

\section{Discussion}

In a similar manner to precipitation, return times for monthly temperatures (not shown) show an increase of temperatures for July in central England and Wales comparing the 2000s ensemble with the decade of 1961-1970 (e.g., Massey et al. 2012). The increase in the odds of a wet July is a credible result given the thermodynamic fact that a warmer atmosphere can contain more water vapour; assuming precipitation extremes are constrained to change with the water vapour-holding capacity of the atmosphere, the Clausius-Clapeyron relation leads to an expectation of increased precipitation, under conditions of constant relative humidity. This argument is typically invoked in the aftermath of floods as an explanation for possible increases in such severe wet events under an anthropogenically warming climate, and is given in more detail in Pall et al. (2011).

Our findings for July corroborate this argument but, since the results for the other two summer months are less clear, we infer that atmospheric dynamics also play an important role. The fact that our analysis of the AMO does not match the observed findings of Sutton and Dong (2012) could be an indication that important processes in the model are not well represented, leading to an inaccurate precipitation signal in the model. However, the mismatch could also indicate that the AMO is not the most important trigger (see also, e.g., Dieppois et al. 2013) of the increased precipitation but that other climatological drivers such as, e.g., the location of the jet-stream, are driving the observed increased precipitation and MSLP anomalies. The less clear, if any, signal for June and August 5-day precipitation indicates that more complex mechanisms than warmer SSTs alone are triggers of changes in summer precipitation in England and Wales. Thus, a logical further study should investigate whether the change in return time of summer precipitation can be associated with a displacement of the jet stream.

\subsection{Implications of uncertainty assessment}

The design of the modelling approach in much of this study aims to quantify the uncertainty in natural variability by simulating large initial condition ensembles very comprehensively; thus our conclusions are unlikely to underestimate this source of uncertainty. However, given the current general lack of skill in simulating precipitation (as opposed to, for example, temperature), the uncertainty in the model structure and parameters have been shown (Fowler et al. 2010) to be of importance for the attribution of extreme precipitation events. The addition of a perturbed parameter ensemble would enable us to quantify more accurately the uncertainty in extreme precipitation 
events, and this represents a promising avenue for further studies which is especially achievable with the climateprediction.net project.

The uncertainty arising from the fact that we do not know how the world might have been without anthropogenic climate change has been addressed by using two different ensembles, an ensemble of the 1960s including all observed forcings (AF1960s) and a counterfactual ensemble of the decade 2000-2010 excluding anthropogenic greenhouse gas forcing by using SSTs with the anthropogenic warming pattern removed (NAT2000s). These two ensembles give some quantification of this uncertainty but most likely this is not comprehensive, as discussed above (Section 2.4). This study could also be furthered, and the uncertainty in its conclusions potentially reduced, if not only the extreme event itself were analysed, but also the weather conditions leading up to the event, and the larger-scale circulation patterns as well. A more detailed description of the flood event in the context of the weather of the spring and summer of 2007, therefore, could well lead to a more accurate analysis of the change in risk of such an event occurring.

\subsubsection{Outcome variable}

The variable quantified in our study is 5-day July precipitation in England and Wales, in the sense that we compare the estimated return period of specific levels of rainfall events in two decades separated by 40 years, and a counterfactual decade. We are able to estimate return periods because of having an extremely large ensemble of different realisations of these two decades (initial condition ensemble).

\subsubsection{Consequence statement}

Results from our study indicate that in the warmer-climate world of the 2000s we might expect 5-day high rainfall events in July to be heavier and occur more frequently, as compared to the 1960s and a counterfactual simulation of the last decade. A change in the pattern of how heavy rainfall events occur has far-reaching consequences for many aspects of society, such as the altered risks of flooding, and changes in water availability due to altered reservoir management needs. In this study we have not made any quantitative estimate of the impacts of such an altered pattern of rainfall events since we have not used our weather event data as input to an impacts model of any kind. Our spread of results could, however, be supplied to river catchment/flood models to indicate the anticipated spread of results in the likelihood of various changes to flood and water availability risks. It is however important to decompose drivers of precipitation to get a clear view of the chain of causality for precipitation changes in England and Wales.

Acknowledgments The authors would like to thank the climateprediction.net participants whose generous donation of their spare computer processing power has enabled such large model ensembles to be created. The authors are also indebted to the paper reviewers for helpful comments that greatly improved the manuscript.

\section{References}

Alexander LV, Jones PD (2001) Updated precipitation series for the U.K. and discussion of recent events. Atmos Sci Lett 1:1530

Allen MR (1999) Do-it-yourself climate prediction. Nature 401:642

Allen MR (2003) Liability for climate change. Nature 421:891-892 
Allen M, Pall P, Stone D, Stott P, Frame D, Min S-K, Nozawa T, Yukimoto S (2007) Scientific challenges in the Attribution of harm to human influence on climate, vol 155. The University of Pennsilvania Law Review, pp 1353-1400

Chatterton J, Viavattene C, Morris J, Penning-Rowsell E, Tapsell S (2010) The costs of the summer 2007 floods in England. Environment Agency, Rio House, Waterside Drive, Aztec West, Almondsbury, Bristol, BS32 4UD. ISBN: 978-1-84911-146-1

Coumou, Rahmstorf (2012) A decade of weather extremes. Nat Clim Chang 2:491-496

Dieppois B, Durand A, Fournier M, Massei N (2013) Links between multidecadal and interdecadal climatic oscillations in the North Atlantic and regional climate variability of northern France and England since the 17th century. J Geophys Res Atmos 118:4359-4372. doi:10.1002/jgrd.50392

Dole R, Hoerling M, Perlwitz J, Eischeid J, Pegion P, Zhang T, Quan X-W, Xu T, Murray D (2011) Was there a basis for anticipating the 2010 Russian heat wave Geophys Res Lett 38:L06702

Eisenmann I, Untersteiner N, Wettlaufer JS (2007) On the reliability of simulated Arctic sea ice in global climate models. Geophys Res Lett 34:L10501

Fowler HJ, Cooley D, Sain SR, Thurston M (2010) Detecting change in UK extreme precipitation using results from the climateprediction.net BBC climate change experiment. Extremes 13:241-267

Fowler HJ, Ekström M, Blenkinsop S, Smith AP (2007) Estimating change in extreme European precipitation using a multi-model ensemble. J Geophys Res - Atmos 112:D18104. doi:10.1029/2007JD008619

Fowler HJ, Ekström M (2009) Multi-model ensemble estimates of climate change impacts on UK seasonal precipitation extremes. Int J Climatol 29(3):385-416

Fowler HJ, Kilsby CG (2003) A regional frequency analysis of United Kingdom extreme rainfall from 1961 to 2000. Int J Climatol 23(11):1313-1334

Gordon C, Cooper C, Senior CA, Banks H, Gregory J, Johns T, Mitchell J, Wood R (2000) The simulation of SST, sea ice extents and ocean heat transports in a version of the Hadley centre coupled model without flux adjustments. Climate Dyn 16:147-168

Hawkins E, Sutton R (2011) The potential to narrow uncertainty in projections of regional precipitation change. Clim Dyn 37:407-418

Hegerl G, Zwiers F, Braconnot P, Gillet N, Luo Y, Marengo J, Nicholls N, Penner J, Stott P (2007) Understanding and attributing climate change. Cambridge University Press

Jones RG, Noguer M, Hassell D, Hudson D, Wilson S, Jenkins G, Mitchell J (2004) Generating high resolution climate change scenarios using PRECIS. Met Office Hadley Centre, Exeter, UK

Kalnay E et al. (1996) The NCEP/NCAR 40-year reanalysis project. Bull Am Meteorol Soc 77:437-471

Kay AL, Crooks SM, Pall P, Stone DA (2011) Attribution of Autumn/Winter 2000 flood risk in England to anthropogenic climate change: a catchment-based study. J Hydrol 406:97-112

Manley G. (1974) Central England temperatures: monthly means 1659 to 1973. Q J Royal Meteorol Soc 100:389-405

Massey N, Aina T, Allen M, Christensen C, Frame D, Goodman D, Kettleborough J, Martin A, Pascoe S, Stainforth D (2006) Data access and analysis with distributed federated data servers in climateprediction.net. Adv Geosci 8:49-56

Massey N, Aina T, Rye C, Otto FEL, Wilson S, Jones RG, Allen MR (2012) Have the odds of warm November temperatures and of cold December temperatures in central England changed Bull Am Meteorol Soc 93:1057-1059

Otto FEL, Massey N, van Oldenborgh GJ, Jones RG, Allen MR (2012) Reconciling two approaches to attribution of the 2010 Russian heat wave. Geophys Res Lett 39:L04702

Pall P, Aina T, Stone DA, Stott PA, Nozawa T, Hilberts AGJ, Lohmann D, Allen MR (2011) Anthropogenic greenhouse gas contribution to flood risk in England and Wales in autumn 2000. Nature 470:382-386

Peterson TC, Hoerling MP, Stott PA, Herring S (eds) (2013) Explaining extreme events of 2012 from a climate perspective, vol 94

Peterson TC, Stott PA, Herring S (eds) (2012) Explaining extreme events of 2011 from a climate perspective, vol 93

Pope VD, Gallani ML, Rowntree PR, Stratton RA (2000) The impact of new physical parameterizations in the Hadley centre model: Hadam3. Climate Dyn 16:123-146

Rahmstorf S, Coumou D (2011) Increase of extreme events in a warming world. PNAS 108:1290517909

Rayner NA, Parker DE, Horton EB, Folland CK, Alexander LV, Rowell DP, Kent EC, Kaplan A (2003) Global analyses of sea surface temperature, sea ice, and night marine air temperature since the late nineteenth century. J Geophys Res 108:4407

Sparrow S, Huntingford C, Massey N, Allen MR (2013) The use of very large atmospheric model ensemble to assess potential anthropogenic influence on the UK summer 2012 high rainfall totals[in Explaining extreme events of 2012 from a climate perspective]. Bull Am Meteorol Soc 94:S36-S41 
Stott PA, Gillett NP, Hegerl GC, Karoly DJ, Stone DA, Zhang X, Zwiers F (2010) Detection and attribution of climate change: a regional perspective. WIREs Clim Chg 1:192-211

Stott PA, Kettleborough JA, Allen MR (2006) Uncertainty in continental-scale temperature predictions. Geophys Res Lett 33:L02708

Stott PA, Stone DA, Allen MR (2004) Human contribution to the European heatwave of 2003. Nature 432:610-614

Sutton RT, Dong B (2012) Atlantic Ocean influence on a shift in European climate in the 1990s. Nature Geosci 5:788-792

UNFCCC report FCCC/SBI/2012/29 (2012) Report on the regional expert meetings on a range of approaches to address loss and damage associated with the adverse effects of climate change, including impacts related to extreme weather events and slow onset events. Subsidiary Body for Implementation Thirtyseventh session Doha, 26 November to 1 December 2012 